\title{
Exploring the competition between variable renewable electricity and a carbon-neutral baseload technology
}

\author{
Emma Jonson $^{1}$ (D) $\cdot$ Christian Azar ${ }^{1} \cdot$ Kristian Lindgren $^{1} \cdot$ Liv Lundberg $^{1}$
}

Received: 4 November 2017 / Accepted: 14 September 2018 / Published online: 4 October 2018

(c) The Author(s) 2018

\begin{abstract}
In this paper we explore the competition between variable renewable energy sources (VRE) and a carbon-neutral baseload technology in the transition to a low-carbon power system. We study a stylized system subject to a gradually increasing carbon tax using an agent-based model where agents are power companies investing in new capacity. The agents make predictions of the profitability of different investment options. Five electricity generating technologies are available in the model: coal, gas, wind, solar PV and a more expensive carbon-neutral baseload technology. We compare the output from our model with a corresponding optimization model. We present two main findings: (1) installed capacity of VRE initially increases with a carbon tax. However, once the carbon tax has reached a certain level the installed capacity of VRE starts to decline due to competition with the stylized carbon-neutral baseload technology. (2) With limited foresight we find that the model underinvests (first 25 years) in wind and then overinvests in wind compared to the optimal solution. The reasons for these dynamic phenomena are explained and an extensive sensitivity analysis is carried out.
\end{abstract}

Keywords Investment decisions - Variable renewables · Wind power - Solar PV . Agent-based modeling · Optimization

Emma Jonson

emma.jonson@chalmers.se

Christian Azar

christian.azar@chalmers.se

Kristian Lindgren

kristian.lindgren@chalmers.se

Liv Lundberg

livl@chalmers.se

1 Department of Space, Earth and Environment, Chalmers University of Technology, 41296 Göteborg, Sweden 


\section{Introduction}

Policy analysis and decisions about energy and environmental issues are often informed by various forms of optimization models that find the least cost optimized pathway, e.g., GET [1], PRIMES [2], TIMES [3] and LIMES [4]. These models implicitly assume that the large number of independent market actors all are rational profit maximizers with full information and perfect foresight. Markets are assumed to always be in equilibrium, which means that transient non-equilibrium phenomena cannot be properly studied. Ex-post modeling shows that cost optimization does not always approximate real-world electricity system transitions, see e.g. [5]. For these reasons, other types of models may be a useful complement to optimization models when studying the transition to a low-carbon power system.

With an agent-based modeling approach several of the assumptions in optimization models can be relaxed. Here we may model (i) actors that operate under uncertainty about future policies, fuel and electricity prices and other actors' investments; (ii) actors that are sufficiently large to exercise market power; (iii) actors that do not necessarily make rational decisions given the information they have; and (iv) actors that may have other objectives than pure profit maximization. For instance, cooperatives may want to invest in solar or wind even if the profit is higher for other technologies. Such assumptions are difficult to include in optimization models. In a world where electric power systems are becoming increasingly complex, agent-based models may serve as computational laboratories for the validation of market design prior to realworld implementation [6].

We have developed an agent-based model (ABM) where agents are power companies investing in the power market. Capacity expansion is driven by the agents' expectations of future profits, which in turn depend on expectations of (1) future carbon prices, (2) demand, and (3) the composition of the future generation mix. (The carbon price, demand and the generation mix together determine the electricity price.) Because of uncertainty and risk, the agents in our model use a higher discount rate than a social optimizer in a risk free setting. For this paper we use the model to study the effect of limited foresight during the transition to a low-carbon power system. The model represents a country with demand and weather conditions as in Germany, and with stylized market conditions.

Generation companies in the power market generally face two types of problems: the investment decision and the production decision [7]. ABMs applied to the power market have previously been used mainly to study strategic bidding behavior on the wholesale electricity market (e.g. [8-10]). ABMs that model capacity expansion are fewer. An example is EMLab-Generation developed at TU Delft described in [11] and [12]. Here agents base their investment decisions on estimates of future prices, the costs of different generation technologies, and possibly other factors such as risk aversion or a preference for specific generation technologies such as renewable energy.

In order to achieve a strong mitigation of greenhouse gas emissions, a transition to a substantially different energy system is needed. The electricity sector in particular has a large potential for decarbonization given technologies such as wind power, solar $\mathrm{PV}$, nuclear, thermal power plants with carbon capture and storage (CCS) and solar thermal coupled with hydrogen storage. These technologies can be divided into two 
main categories: the first is variable renewable energy sources (VREs), which include wind and solar power. The output from these technologies is intermittent since it is determined by weather. The second category includes carbon-neutral technologies with baseload characteristics such as nuclear, thermal power plants with CCS and solar thermal coupled with hydrogen storage. They have high investment costs but relatively low variable operating costs and their economic model is based on them selling electricity during most hours of the year. These technologies are also "dispatchable", meaning that they can adjust output in response to demand.

The aim of this paper is to analyze how VREs, i.e., non-dispatchable technologies, compete with conventional fossil fuels (gas and coal) and dispatchable carbon-neutral technologies under increasing carbon taxes. Hirth [13] analysed the optimal wind deployment in steady state for various carbon taxes. A similar study has been done by de Sisternes et al. [14]. In our paper, we take this analysis one step further by analyzing the transient non-equilibrium development of the electricity system under increasing carbon taxes using an ABM. In addition, we compare our result with those obtained using an optimization model and analyze why the models give similar but somewhat different results. In addition, we find a couple of properties of the behavior of wind and solar when competing against fossil and carbon-neutral dispatchable electricity technologies, that also Hirth and de Sisternes et al. have noted, and we analyze and explain these features and the mechanisms behind them further.

The paper is structured as follows. In Sect. 2 we describe our ABM. Section 3 declares the data used. Section 4 describes our results. In Sect. 5 we discuss our results and Sect. 6 concludes.

\section{Methodology}

Agent-based modeling is, in our perspective, a mathematical modeling approach in which decisions serving as driving mechanisms in the time development of the system are explicitly represented. In the literature it is often said to be a computational method that enables a researcher to create, analyze, and experiment with models composed of agents that interact within an environment [15]. In these models individual entities (the agents) and their interactions are directly represented and the agents' decision rules are explicitly specified. The ABM presented in this paper is designed to study the electricity system and to capture both investments in power plants made over time and output (dispatch). ABMs have been used in different areas of economics [16] and calls are being heard to increasingly use this type of modeling to better estimate the costs of climate change mitigation [17]. We believe that this modeling approach can play an important role in the study of power system transitions. The ABM presented in this paper is a first step in our attempt towards a more developed representation of investors in the power market. The agents in our model are power companies investing in new capacity. They are characterized by their beliefs about the future power system and future carbon tax. The model runs in 1-year steps.

We model a stylized power system with one single node. Trade of electricity with other regions and electricity storage are not included in the present version. Variability of supply and demand is explicitly represented as time slices over the year, with each 
time slice characterized by a certain wind and solar availability as well as a certain shift in the demand schedule. The model horizon is 70 years and we have no stochasticity in the model.

\subsection{Supply and demand}

We assume an ideal electricity market in the sense that neither producers nor consumers make strategic bids to affect the market price. Power plants are dispatched according to the merit order (i.e., according to marginal cost of production) and each hour a price is determined that clears the market. We model demand as iso-elastic, so that the relation between the delivered and consumed power $q_{\tau}$ and corresponding price $p_{\tau}$ in time slice $\tau$ is

$$
q_{\tau}=q_{0, \tau}\left(\frac{p_{\tau}}{p_{0}}\right)^{\epsilon}
$$

where $\epsilon$ is the short term price elasticity of demand and $p_{0}$ is a constant. We use elasticity $\epsilon=-0.05$ and $p_{0}=3.25$ cent $/ \mathrm{kWh}$. The "demand reference level" $q_{0, \tau}$ is a parameter that shifts the demand curve in each time slice reflecting the variations of demand over seasons and times of day. In this version of the study we utilize German hourly demand levels from year 2011 as a proxy for $q_{0, \tau}$. This is a simplification, since a low (high) demand level in a certain hour may have been the result of a high (low) price in that hour, rather than a change in consumer preferences.

In each year the power system is characterized by the installed capacity of the different power technologies together with the age profile of the plants. For each time slice the model finds the price that clears the market, i.e., the price at when demand equals supply. The last dispatched plant may operate at a fraction of its full capacity. In this model, the plants with the highest variable operating cost must rely on hours of scarcity to make sufficient revenues to pay back the investment costs. In reality plant owners may use market power to affect prices even during hours when demand is below total capacity, if the excess capacity is sufficiently small [18]. This option is not allowed here.

The year is divided into time slices where each slice $\tau$ is characterized by demand reference level $q_{0, \tau}$, wind power output and solar power output as the shares of installed capacity. Appendix A describes the time slicing further. In this study we let the time slicing representation of the year be constant over the modeling horizon (i.e., no change in demand reference level or solar and wind patterns from one year to another). We ignore any operational considerations such as minimum up and down times, ramp rates and part-load performance, which allows us to disregard any chronology within a year. (Costs associated to start-ups and shutdowns are also ignored.) Finally we disregard must-run requirements of conventional power plants necessary for grid services like voltage stabilization etc.

We assume that plants start to operate the year after the investment decision is made and the investment cost is paid. Each plant produces electricity whenever the electricity price is higher than or equal to the variable operating cost. The model starts 
at time $t=0$ with an equilibrium capacity of power plants, given a carbon tax of 0 $\$ / \mathrm{tCO}_{2}$, and with a uniform distribution of remaining plant lifetimes.

\subsection{The investment decision}

Investment decisions are made as follows. Each year the agents form expectations about future installed capacity, the future demand and the future carbon tax. Based on these expectations the agents calculate the future electricity price if a new investment were to be made in each type of power plant. The predicted electricity price is then used to assess the net present value $(N P V)$ of each investment option. In this study we let agents use a discount rate of $8 \% / y e a r$. This rate is chosen to represent the long term return on capital corrected for risk that an agent investing in power plants would typically assume. In the power market each plant type has its own risk profile that is also strongly affected by the overall capacity mix of the market [19]. The discount rate used typically also varies with type of investor and country. See e.g. [20,21] for more details. For simplicity we assume that all actors have the same discount rate for all investment options.

We assume that agents (companies) are profit maximizers and that in order to achieve that goal, they rank projects based on their profitability index $(P I),{ }^{1}$ i.e., the NPV of future net revenues divided by the investment cost. In addition, since some types of power plants have significantly longer life times than others, we have corrected for this by looking at the expected annuitized net revenues (per euro invested), i.e., the highest

$$
C R F * P I
$$

where

$$
C R F=\frac{r(1+r)^{T}}{(1+r)^{T}-1}
$$

is the capital recovery factor, $T$ is the lifetime of the project and $r$ is the discount rate. ${ }^{2}$

We assume in this model that agents know how much installed capacity there is of each power plant type, which plants will be decommissioned during the year, and how many new plants that currently are being invested in by other agents. Agents also know the carbon tax and the level of demand for the following year. Agents therefore have relatively detailed information about the conditions of the power system the following year, but no additional knowledge beyond that. For this study, agents assume that the conditions of the following year will hold into the future.

\footnotetext{
1 When funds are limited companies maximize profits by prioritizing the projects with the highest profitability index. Although few companies face capital rationing in absolute terms many of them have self imposed capital constraints. Reasons for this are that companies want to limit their level of indebtedness and also want to avoid problems from growing too fast. Thus, ranking projects by their profitability index is a common capital budgeting technique in industry [22].

2 As a sensitivity analysis we tested other ranking criteria, e.g. NPV, and the results were very similar.
} 
We have implemented the model in the following way. In a given year agents are first randomly selected to make investments in new power plants, which they do if there are plants that they expect to be profitable. Information about the investment is made public to the other agents, which means that subsequent investment decisions take into account previous decisions in the same year. When no more agents want to invest, power plants that have reached the end of their lifetimes are decommissioned one at a time. The agents assume that the plants that have not yet been decommissioned will continue to operate in the following year. After each decommissioning the agents make a new prediction and invest in new power capacity if they want to. Plants start to produce electricity at the beginning of the following year. The chosen implementation guarantees that the agents make a correct estimation of future prices as long as the model remains in a steady state over time.

Different types of power plants generally differ in size. For instance, nuclear power plants typically have higher capacity than wind power parks. At the same time not all power plants of the same type are equally large. A $1000 \mathrm{MW}$ nuclear power plant may have a positive NPV while a $1100 \mathrm{MW}$ plant is assessed to be unprofitable. In this implementation we let agents invest in power plant modules of just $50 \mathrm{MW}$ at a time, in order to not disfavor technologies with large plant sizes and to limit the effect that each investment has on prices. The resulting total investments, after a year of accumulated decisions, typically correspond to plants larger than the module value.

In this first study agents do not take their existing portfolios into account when making an investment. They only consider the new investment opportunity in isolation. They are also homogeneous in their strategies. This means that the exact number of agents is insignificant for our analysis. If any new power plant is expected to be profitable, one of the agents will make the investment. Agents are not allowed to change their minds once an investment decision is taken.

\subsection{The optimization model}

The ABM will be compared with an optimization approach that finds the optimal path of investments assuming full information of all aspects of the system for the full time period. The optimization model is a standard non-linear optimization based on maximization of total surplus of consumers and producers, thus reflecting a market equilibrium solution of the transition without uncertainty. The details of the optimization model implementation is presented in Appendix B. The optimization model represents the same stylized power system, and with the same supply and demand characteristics, as the ABM. If the same discount rate is used the two models find the same steady state.

\section{Data}

We have chosen to apply our model to a power system resembling Germany in terms of demand as well as solar and wind potential. Data on wind speeds and solar insolation 
Table 1 Parameter values

\begin{tabular}{llllll}
\hline & Coal & Gas & Solar PV & Wind & CN-Baseload \\
\hline $\begin{array}{l}\text { Investment costs }(€ / \mathrm{kW}) \\
\begin{array}{l}\text { Variable operating costs } \\
\text { a }\end{array}\end{array}$ & 1450 & 900 & 800 & 1500 & 6000 \\
$\begin{array}{l}\left.\text { cent } / \mathrm{kWh}_{e l}\right) \\
\text { Emissions intensity }\end{array}$ & 2.0 & 4.5 & 0 & 0 & 1.0 \\
$\quad\left(\mathrm{~g} \mathrm{CO}_{2} / \mathrm{kWh}_{e l}\right)$ & 1000 & 450 & 0 & & \\
Lifetime (years) & 40 & 30 & 25 & 25 & 40 \\
$\mathrm{LCOE}^{\mathrm{b}} \quad\left(\right.$ cent $\left./ \mathrm{kWh}_{e l}\right)$ & 3.7 & 5.6 & 7.1 & 5.0 & 8.2 \\
\hline
\end{tabular}

${ }^{\mathrm{a}}$ Excluding the carbon tax

${ }^{b}$ Levelized cost of electricity, assuming a load factor of $80 \%$ for the dispatchable power technologies and a discount rate of $8 \% / y e a r$

were taken from the European Centre for Medium-Range Weather Forecasts ${ }^{3}$ with a temporal resolution of three hours. We use data from year 2008, which is a year with close to average wind speeds in Northern Europe. The wind speed is taken from level 59 corresponding to a hub height of approximately 93 meters. The wind speed data was processed by applying an output function simulating state-of-the-art wind power plants, as described in [23]. The solar insolation data was processed with an output function simulating fixed silicon solar cells, as described in [24]. We assume that wind power plants and solar cells are distributed homogeneously over the country, i.e., we do not give priority to sites with above-average wind conditions. The modeled capacity factors of the wind and solar PV fleets are $32 \%$ and $12 \%$, respectively. This can be compared with the observed capacity factor of German on-shore wind power in 2015 , which was $22.7 \%$ [25]. The low capacity factor can be attributed to older plants having shorter towers, shorter blades and comparatively larger generators than modern ones. Many countries do already today have wind power capacity factors above $32 \%$, e.g. Ireland, Norway and the United States [25]. The electricity demand data are for year 2011, which is a typical year in terms of demand, obtained from ENTSO-E. ${ }^{4}$

\subsection{Scenario and parameter values}

Five types of power plants are included: coal-fired power plants, natural gas fired plants, solar photovoltaic (PV) systems, wind power plants and a generic carbonneutral baseload technology (CN-Baseload). Each type of power plant is characterized by an investment cost, a variable operating cost, a $\mathrm{CO}_{2}$ emission intensity and a fixed plant lifetime (see Table 1). The model is initiated with a fleet of power plants corresponding to the equilibrium capacity in our system without a carbon tax. The model is run with a pre-defined carbon tax trajectory (see Fig. 1) where a carbon tax is introduced after 10 years starting at $0 € /$ ton $\mathrm{CO}_{2}$ and increasing linearly up to 100 $€ /$ ton $\mathrm{CO}_{2}$ in year 50. The tax remains at that level until year 70. In the EU there

\footnotetext{
3 http://www.ecmwf.int

4 https://www.entsoe.eu/data/data-portal/consumption/
} 


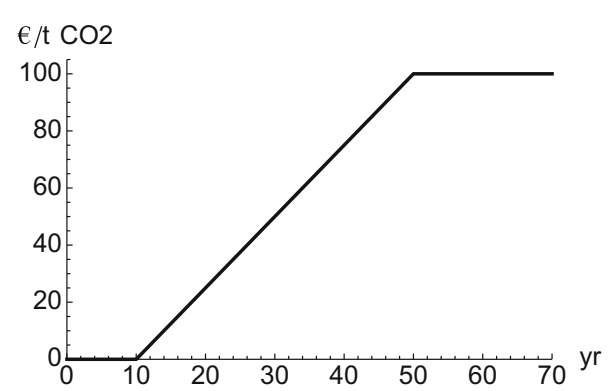

Fig. 1 The carbon tax trajectory

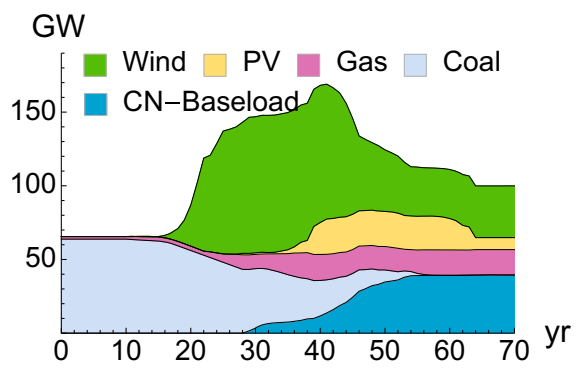

(a) Installed capacity

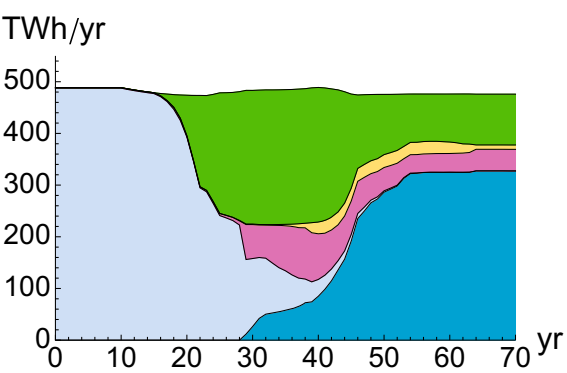

(b) Electricity produced

Fig. 2 Installed capacity by year (a) and electricity produced (b) in the ABM. a Installed capacity. b Electricity produced

is already a price on $\mathrm{CO}_{2}$ emissions, but it is low. Given the current high supply of emission rights, it may take a decade before the price of $\mathrm{CO}_{2}$ emissions increases.

\section{Results}

\subsection{Electricity generation and installed capacity}

The aim of this paper is to explore how VRE and a carbon-free baseload power technology compete in the transition to a low-carbon power system. Figure 2 shows the installed capacity over time and the annual electricity generation in our model. The first years, when there is no carbon tax, our power system is dominated by coal-fired power plants and complemented with a small amount of gas as peak capacity. When the carbon tax is introduced both VRE and CN-Baseload gain a relative advantage compared to coal fired plants, and compete with each other for room in the energy mix as the tax continuously increases. Wind power is the first carbon-neutral technology that is introduced after the adoption of the carbon tax. As the carbon tax continues to increase $\mathrm{CN}$-Baseload and solar PV eventually start growing while wind declines. This happens despite the fact that $\mathrm{CN}$-Baseload has a higher LCOE than wind. At the end of the model run also the installed capacity of solar PV declines. When the carbon tax stabilizes the installed capacity of the different technologies eventually reach their steady state levels. 
Fig. 3 The capacity factors of CN-Baseload, coal and gas

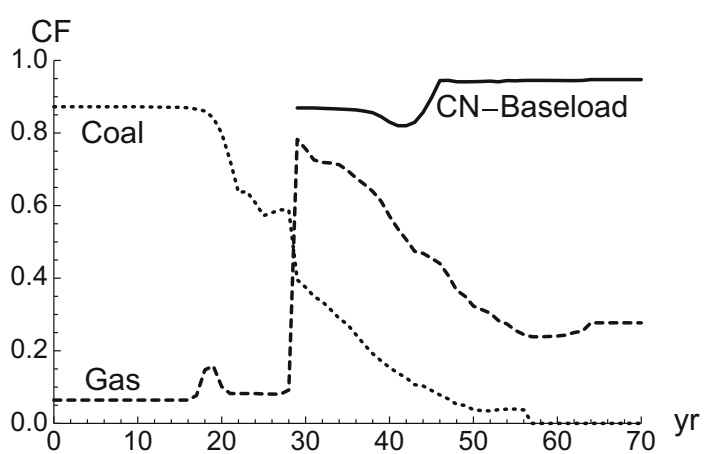

The capacity factors of CN-Baseload, coal and gas are shown in Fig. 3. CN-Baseload has a low variable operating cost and appears early in the merit order. Its capacity factor is always high, in the range of $82-95 \%$. In this model we let demand be elastic and we have no curtailment of VREs. The capacity factors of solar PV and wind therefore lie at constant levels over the years, at $32 \%$ and $12 \%$, respectively.

For gas and coal the story is different. In year 30 we can see a sudden dramatic increase in the electricity generation of gas. This technology starts out as peak capacity running only a few hundred hours per year but then increases its capacity factor to around $80 \%$. At the same time coal decreases its electricity generation. The reason is that coal and gas switch places in the merit order when the tax is sufficiently large. From year 30 the capacity factors of both coal and gas decrease continuously as the carbon tax grows. The reason is the expansion of VREs and CN-Baseload that appear first in the merit order.

\subsection{The electricity price}

Figure 4 shows the development of the electricity price over time. The average yearly electricity price increases when the carbon tax is introduced (see Fig. 4a). The reason is that the carbon tax increases the variable operating costs of coal and gas, which raises the electricity price during the hours when these power sources set the electricity price. It should be noted that the electricity price does not increase in line with the carbon tax after a few decades. The higher cost for fossil power leads to an increasing share of VRE and CN-Baseload in the electricity system, and the cost of these technologies does not increase with the carbon tax, i.e., the price increase expected from the increase in the carbon tax is mitigated by the increasing use of VRE and CN-Baseload. We also have a shift towards more hours with gas on the margin instead of coal. Since the former technology is less carbon intensive than the latter, the average electricity price becomes less affected by the carbon tax.

Figure $4 \mathrm{~b}$ shows the average revenues received by $\mathrm{CN}$-Baseload, solar $\mathrm{PV}$ and wind. We note that the average revenues differ by technology. This is explained by the fact that different technologies generate electricity at different points in time and that the electricity price varies over time. In particular we note that the average revenues per $\mathrm{kWh}$ received by wind power remain at a comparatively low level even as the average 


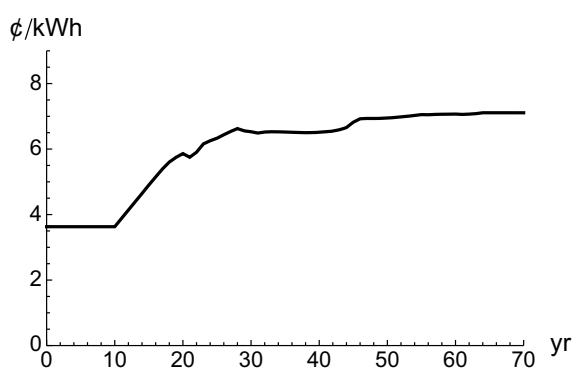

(a) Average electricity price

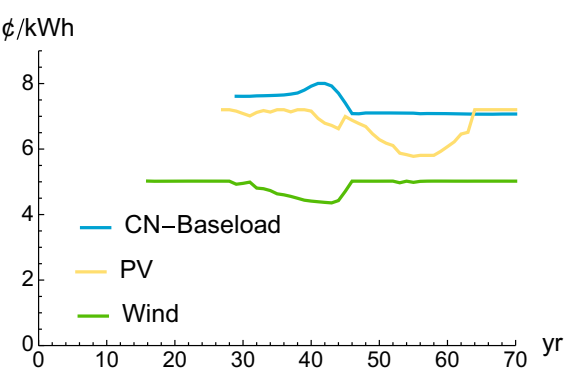

(b) Average revenues by technology

Fig. 4 a Average electricity price by year and $\mathbf{b}$ average revenues from sold electricity by power technology and year. a Average electricity price. $\mathbf{b}$ Average revenues by technology

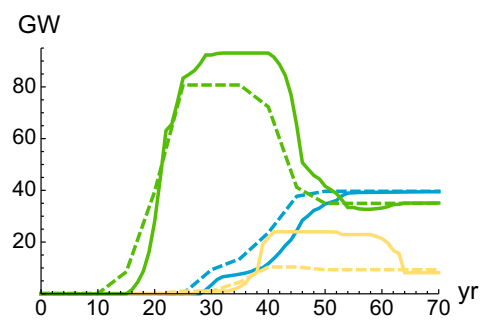

- CN-Baseload ABM

-- CN-Baseload Opt

- PV ABM

-- PV Opt

- Wind ABM

-- Wind Opt

Fig. 5 Installed capacity by year of CN-Baseload, solar PV and wind, in the ABM and the corresponding optimization model

electricity price rises. This can be explained in the following way. Agents in the model invest in wind (or any technology) as long as there is profit to be made, i.e., the net present value of all revenues exceed total costs including the investment costs. This means that if the average revenue received per $\mathrm{kWh}$ of electricity sold by the wind operators is higher than the LCOE for wind power, agents will invest in more wind since there is profit to be made. When that is done, the average revenue drops since the price of electricity will drop the more wind there is in the market. Investments in wind will stop when the average revenue drops below the LCOE for wind. Thus, for that reason the average price of electricity received by wind operators will hoover close to the LCOE of wind. The reason for the drop in the revenues received (during the years 30-45) has to do with transient non-equilibrium phenomena due to limited foresight of the agents. The same reasoning applies to solar PV and other technologies.

\subsection{Comparison with an optimization model}

How does limited foresight affect the competition between $V R E$ and a carbon-neutral baseload technology such as nuclear? To answer that question we compare what happens in the ABM with our optimization model of the same electricity system.

In Fig. 5 we show the installed capacities of $\mathrm{CN}$-Baseload, wind power and solar $\mathrm{PV}$ in the ABM and the optimization model when both models have the same discount rate of $8 \%$ /year. The installed capacity of wind is lower in the ABM than in the optimal 


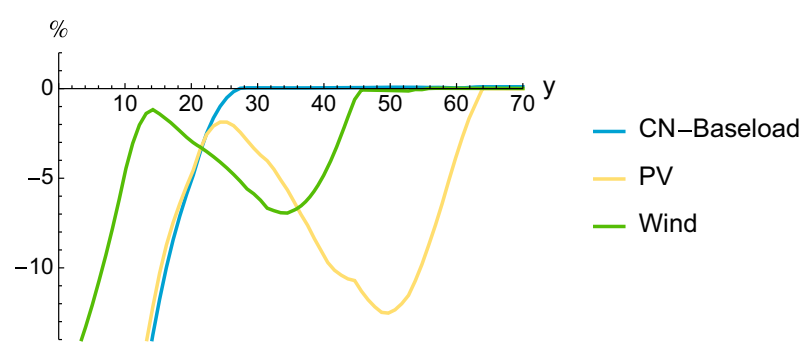

Fig. 6 Ex-post profitability index of CN-Baseload, solar PV and wind power in the ABM as a function of the year of investment. (The figure shows the profitability index for all years, not only the years when investments actually were made.) The profitability index is calculated with a discount rate of $8 \% / y e a r$

solution during the first years after the carbon tax is introduced. Between years 25 and 50 this relationship is reversed. Both of these observations can be explained by the agents' limited foresight. In the first years the agents are ignorant of the increase in the carbon tax which means that they underinvest in wind power. In the later years they fail to anticipate the subsequent introduction of $\mathrm{CN}$-Baseload in the power system and therefore over-invest in wind.

The installed capacity of CN-Baseload is lower in the ABM than in the optimization model in the transient phase. Due to the over expansion of wind power from year 25 there is less room for $\mathrm{CN}$-Baseload to expand profitably in the ABM (prices become depressed). We also note that the ABM invests more in solar PV in years 35-40 than what is given by the optimal solution. Less installed capacity of $\mathrm{CN}$-Baseload makes it possible for solar PV to expand more. At the end of the model run when the carbon tax has stabilized, the two models eventually find the same steady state.

Figure 6 shows the ex-post profitability index of the ABM as a function of the year the investments are made, calculated with a discount rate of $8 \%$ /year. We can see that the profitability index of wind ends up well below zero during most of the model run, except in the final years. We note that even though the installed capacity of wind is lower than the optimal solution in years 15-20, the profitability index is still negative during these years. The reason is the subsequent over-investment in wind power, which reduces the revenues also for the wind power plants that were built earlier. In summary, the over-investment of wind (compared to the optimal solution) reduces the competitiveness of $\mathrm{CN}$-Baseload, but also decreases its own profits by expanding "too much".

Figure 7 shows the $\mathrm{CO}_{2}$ emission trajectory for three model runs (an $\mathrm{ABM}$ run with a discount rate of $8 \% / y e a r$ and two runs with the optimization model, one with a discount rate of $4 \%$ /year and one with a discount rate of $8 \%$ /year). We can see that the emissions are slightly higher in the ABM than in the optimization model with $r$ $=8 \% /$ year (i.e., for the same discount rate). The reason for this is that the carbonneutral technologies are introduced earlier in the optimization model than in the ABM since there is perfect foresight in the optimization model which means that the ever increasing carbon tax is taken into account in the investment decision in the model. Between years 10 and 35 there is also more gas in the former model than in the latter. It is also interesting to compare with the optimization model with a discount rate of only 
Fig. 7 Yearly emissions for the ABM and the optimization model with a discount rate of $8 \% /$ year (Opt8) and 4\%/year (Opt4)

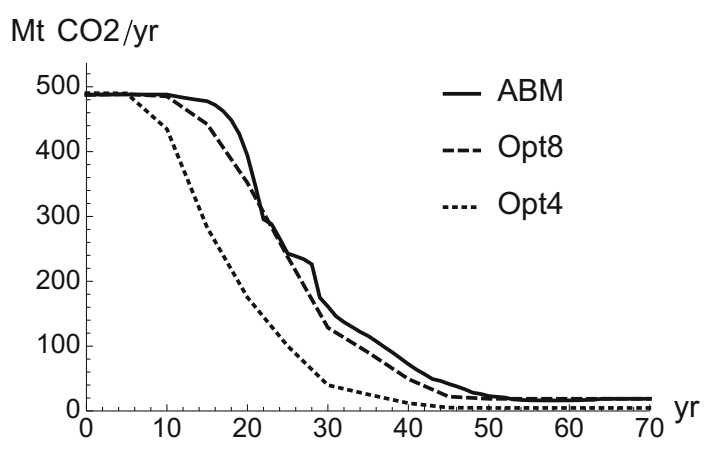

4\%/year (Opt4). In this case the annual emissions are significantly lower, since the lower discount rate tends to benefit technologies with a higher investment cost (like solar, wind and nuclear). Since optimization models typically use the risk free social rate of discount (which is lower than private discount rates) they likely overestimate the $\mathrm{CO}_{2}$ reduction that would take place in the real world as a consequence of a carbon tax. Although the mechanism behind this is well understood this (likely) underestimation of the required carbon tax is rarely pointed out when results from optimization models are presented. Two recent exception are Iyer et al. [26] and Hirth and Steckel [27].

Both the ABM and the optimization model describe the development of a power system over time as it undergoes a transition. The models are generally not in steady state in any particular time step, except in the initial and final years when all costs are constant over time. In Appendix C we estimate the steady state capacities for each tax level for comparison.

\subsection{Sensitivity analysis}

\subsubsection{The role of gas}

In this section we examine the sensitivity of our results with respect to the price of natural gas. Figure 8 shows the installed capacity of CN-Baseload and wind for three different levels of the gas price: $3.5 \mathrm{cent} / \mathrm{kWh}, 4.5 \mathrm{cent} / \mathrm{kWh}$ (baseline) and infinity (a case where gas is not available). As before we see a "rise and decline" behavior of wind when the system is exposed to an increasing carbon tax. We also note that the final installed capacity of wind, which might be surprising, is higher the lower the price of gas. The reverse is true for $\mathrm{CN}$-Baseload.

\subsubsection{A scenario with favorable conditions for solar PV}

In this section we examine how solar PV responds to a carbon tax when it is the sole source of VRE, and the meteorological conditions for solar power are more favorable. We do this by running our ABM again in the absence of wind power and with higher solar insolation. For this analysis we increase the capacity factor of solar PV with $50 \%$, which roughly corresponds to the conditions in countries such as Israel and 


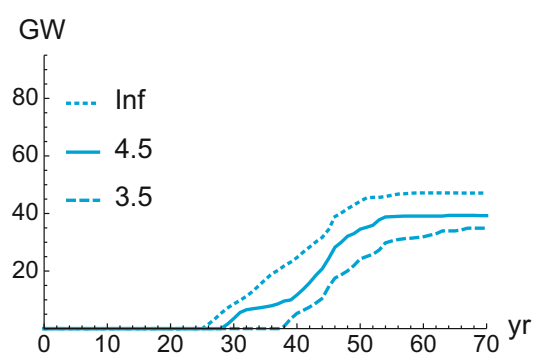

(a) CN-Baseload

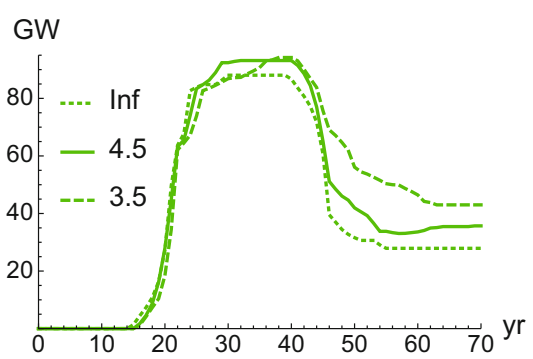

(b) Wind

Fig. 8 Installed capacity of CN-Baseload (a) and wind (b). The figure illustrates three scenarios: a gas price of 3.5 cent $/ \mathrm{kWh}, 4.5$ cent/kWh (baseline) and infinity. a CN-Baseload. b Wind

Fig. 9 Installed capacity of $\mathrm{CN}-$ Baseload and solar PV in the $\mathrm{ABM}$ when we omit wind power and increase the capacity factor of solar PV with $50 \%$

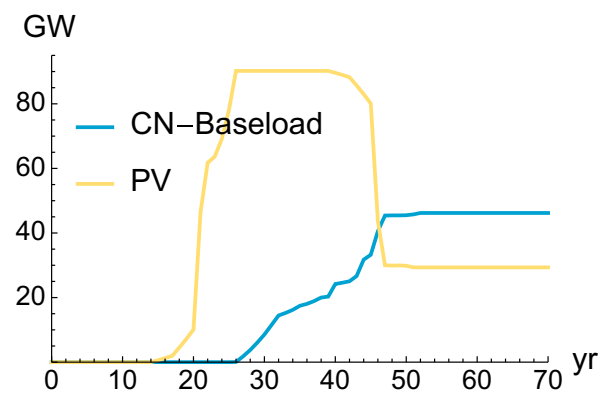

Mexico. Figure 9 shows the installed capacity of CN-Baseload and solar PV under these conditions. We can see that the installed capacity of solar PV first increases but eventually declines again, whereas CN-Baseload continues to grow. Solar PV is in this scenario around four times higher than in our base case at the end of the model horizons.

\subsubsection{A carbon-neutral dispatchable technology with peak load characteristics}

Our generic carbon-neutral baseload technology (CN-Baseload) has a high investment $\operatorname{cost}(6000 € / \mathrm{kW})$ and low variable operating cost $(1 \mathrm{cent} / \mathrm{kWh} /)$. We now study a scenario where this technology is replaced by a different carbon-neutral technology with a low investment cost $(350 € / \mathrm{kW})$ and high variable operating cost $\left(6.3\right.$ cent $\left./ \mathrm{kWh}_{e l}\right)$. The lifetime is still 40 years. We call this technology CN-Dispatchable.

Figure 10 shows the installed capacities by year in our model in this " $\mathrm{CN}$ Dispatchable scenario". Figure 11 compares the installed capacity of wind and the generic carbon-neutral technology to the baseline scenario. We see that when $\mathrm{CN}$ Baseload is replaced by CN-Dispatchable the "rise and decline" of wind disappears.

The installed capacity of CN-Dispatchable in year 70 is larger than that of $\mathrm{CN}$ Baseload in the baseline scenario (as seen in Fig. 11) but the electric output is smaller. The capacity factor of CN-Dispatchable at the end of the model horizon is only $50 \%$ while it is $95 \%$ for CN-Baseload. Given these capacity factors the LCOE is 7.1 cent/kWh for $\mathrm{CN}$-Baseload but only 7.0 cent/kWh for $\mathrm{CN}$-Dispatchable. We can 
Fig. 10 Installed capacity by year

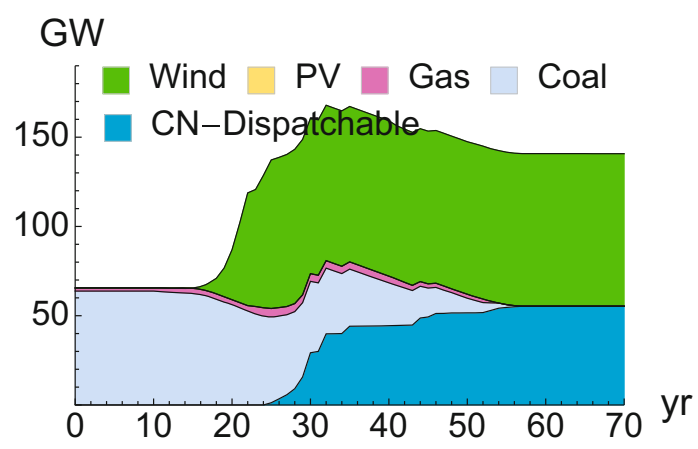

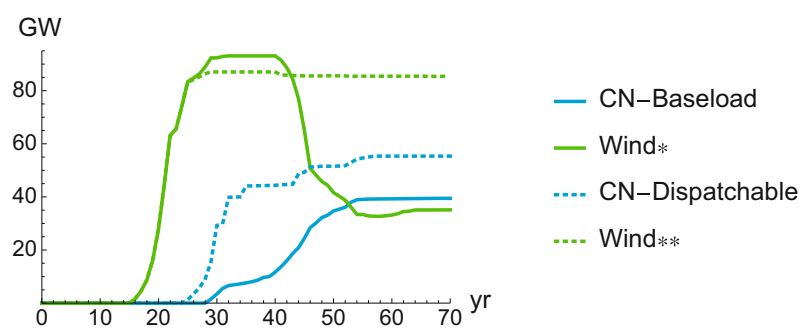

Fig. 11 Installed capacity in our baseline scenario (CN-Baseload and Wind*) and in the CN-Dispatchable scenario (CN-Dispatchable and Wind**)

conclude from this that wind is able to gain a significantly larger market share when it competes against $\mathrm{CN}$-Dispatchable than when it competes against $\mathrm{CN}$-Baseload, even though CN-Dispatchable has a lower LCOE than CN-Baseload.

\subsubsection{A social risk-free rate of discount}

The agents in our ABM use a discount rate of $8 \% / y e a r$. This is higher than what is typically assumed in optimization models. In these models the discount rate is often chosen with the aim of representing the preferences of society as a whole under riskfree conditions. In this section of the sensitivity analysis we let the agents use a value of $4 \% / y e a r$. This value represents the social risk-free rate of discount and lies within the ranges of estimates using both descriptive and normative approaches [28-30].

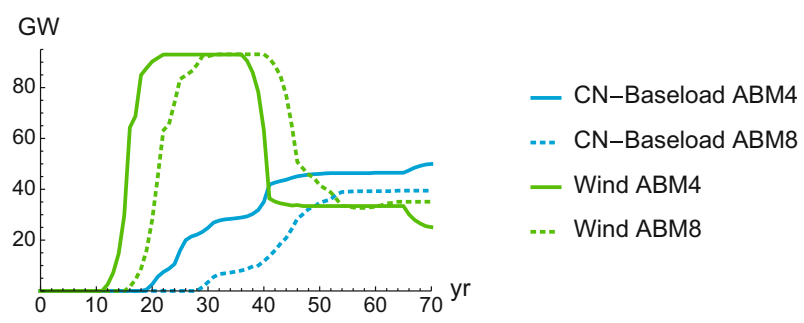

Fig. 12 Installed capacity by year of wind, PV and CN-Baseload when agents use a discount rate of $4 \%$ year (ABM4) and 8\%/year (ABM8) 
Figure 12 shows the installed capacities by year of $\mathrm{CN}$-Baseload and wind. We can see that wind still shows a "rise and decline" behavior as the system is exposed to an increasing carbon tax. We compare the output with the original model run with a discount rate of 8\%/year (ABM8) and make two observations: first, the carbonfree technologies are introduced earlier. The reason for this is that CN-Baseload and wind are very capital intensive technologies, which means that the price drop from a lower discount rate is larger than for other technologies. Second, the final capacity of $\mathrm{CN}-\mathrm{Baseload}$ increases with a lower discount rate, while the reverse is true for wind. $\mathrm{CN}-\mathrm{Baseload}$ is more capital intensive than wind and therefore increases its market share at the expense of wind when these two technologies are the main competitors.

\section{Discussion}

\subsection{Why does the wind power capacity decline with very high carbon taxes?}

It is interesting to note that the installed capacity of wind first increases with a carbon tax and then decreases. The basic mechanism behind this can be explained in the following way. In Fig. 13 we display the levelized cost of generating electricity (LCOE) over time for the main technologies in this analysis (with data from Table 1).

Initially, when the carbon tax is zero or very low, coal is the most competitive technology (see Fig. 13) and the electricity system is also dominated by coal. As the carbon tax increases, coal becomes more expensive and by the year 15-20, wind is beating coal (with this measure). In Fig. 2a we see that wind starts to expand rapidly behind this point in time

However, with the carbon tax level that is reached around the year 30, coal also becomes more expensive than $\mathrm{CN}$-Baseload. This means that there will be no more investment in coal, because coal is beaten by both wind and CN-Baseload. We can thus see in Fig. 2a that CN-Baseload increases over the period 30-55.

But here an interesting question arises. Why is it that $\mathrm{CN}$-Baseload also beats wind (at least partly) for the carbon taxes that prevail beyond the year 30 even though the LCOE of CN-Baseload is significantly higher than that of wind? (As the carbon tax increases and $\mathrm{CN}$-Baseload expands, the installed capacity of wind is reduced to less

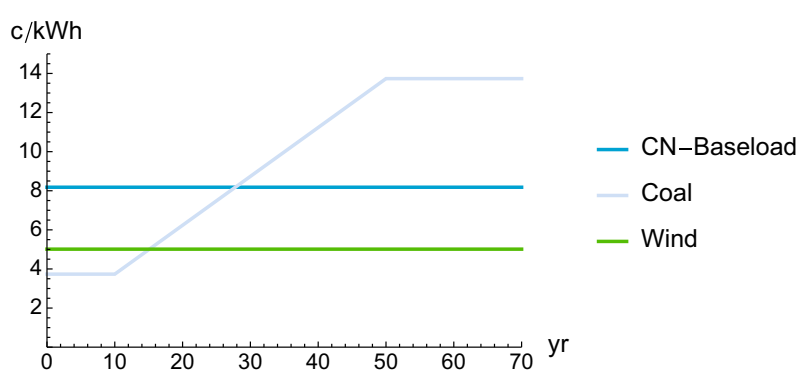

Fig. 13 LCOE for coal, wind and CN-Baseload as a function of time. The change in LCOE is for the sake of simplicity and clarity only driven by the increase in the carbon tax 
than half of what it was at its peak.) The reason for this has to do with the fact that wind is an intermittent technology.

In an electricity system, there is demand for electricity all the time, but wind is an intermittent technology. Wind without storage (or a lot of trade with other regions) cannot meet demand at all points in time so market incentives will trigger investments in dispatchable technologies. In our model investments will be made in $\mathrm{CN}$-Baseload when the carbon tax is sufficiently high. Once CN-Baseload is expanded, it is cheaper to run those plants than to build wind power plants, i.e., the variable operating cost of CN-Baseload is lower than the LCOE of wind. For that reason, CN-Baseload takes most of the market once the carbon tax makes coal (and gas) too expensive.

Finally, our results and analysis illustrate that LCOE is sometimes a poor measure of comparing the competitiveness of electricity generating technologies. The reason for this is that LCOE only refers to the cost of generating the electricity and disregards from the revenues. For technologies that generate electricity at the same time, this is not a problem since their average revenues will be the same. Under these circumstances LCOE is useful as seen here in our analysis of what happens in our ABM when CNBaseload starts to compete with coal. But for wind, and in particular solar, the average revenues may differ substantially from the average price of electricity, in particular when large amounts of wind and solar are put in place in the electricity system. Under these conditions, LCOE is not a good measure of the competitiveness of the different technologies. We see here in our model analysis that investments are made in CNBaseload despite the fact that its LCOE is higher than that of wind (and solar).

So far, we have explained why CN-Baseload expands at the expense of wind despite the fact that it is (according to LCOE) a more costly technology than wind. Now, we also need to explain why wind takes a larger share of the electricity market around the years 25-30 than around the year 50, i.e., when it competes primarily against coal than when its primary competitor is CN-Baseload.

This has to do with the fact that the variable operating cost for coal (with the carbon $\operatorname{tax}$ ) is much higher than the variable operating cost of CN-Baseload and that the electricity price is set by the variable cost of the marginal power technology in the system. (The exception is when generating capacity is scarce and prices are pushed up even higher.) When there is a lot of coal in the market and the carbon tax is relatively high (e.g., years 25-30), wind is more competitive than when coal has been pushed out by $\mathrm{CN}$-Baseload. For that reason the optimal level of wind drops once $\mathrm{CN}$-Baseload enters the market (becomes competitive over coal and gas). However, if $\mathrm{CN}$-Baseload is replaced by a different carbon-neutral dispatchable technology with a high variable operating cost (i.e., CN-Dispatchable in Sect. 4.4.3) wind may keep its competitiveness.

Thus, we can make the following more general observation. Assume that an intermittent technology like wind, competes in an electricity system with either a dispatchable technology A or a dispatchable technology B. Assume further that technologies A and B have the same ex ante estimated levelized cost of electricity (given a certain load factor) but that A has lower variable cost and higher investment cost than B (i.e., technology A could be nuclear and B could be a biogas fueled combined cycle power plant). Still, the resulting share of wind when allowed to compete against either 
of these technologies, will be higher or even much higher when competing against B than competing against $\mathrm{A}$.

\subsection{The electricity price}

In Sect. 4.2 we show how the electricity price develops over time in our model. We find that the price rises as the carbon tax increases. It is interesting to compare this result with other empirical and modeling studies of how the expansion of VREs impacts the electricity price, e.g., [31-39].

In these studies, the price of electricity drops with the expansion of VRE. It drops both on average and, more significantly, during the hours when wind and solar generates most of its electricity. The reason for this is that solar and wind have low (or close to zero) running costs and decrease the price through the merit order effect (see e.g. Hirth [40]). The studies cited here concern markets where wind and solar are introduced through a subsidy scheme. It is important to keep in mind that a lower electricity price does not necessarily imply lower total costs for the consumer since the subsidy is paid via a levy on top of the electricity price or by the government.

In our study we assume that there is no subsidy and that the only policy incentive to reduce emissions is the carbon tax. Under these conditions, there is a relative drop in the electricity price compared to the increase that otherwise would have been expected without VREs. However, there is no absolute drop in the electricity price as a result of the introduction of VRE as is the case in many countries today that introduce VRE through subsidy scheme. This distinction may be important to keep in mind.

\subsection{Limited foresight of agents}

In this paper we use our ABM to study the effect of limited foresight during the transition to a low-carbon power system. Limited foresight in energy systems models is not new, see e.g. [41-44]. The motivation for such an assumption is, as often stated, to better simulate market behavior, in comparison with optimization models, and the decision environment under which decision makers must operate. In future work we intend to study different learning mechanisms and how they affect a transition of the power system.

\section{Conclusions}

In this study we present an agent-based model (ABM) of a dynamic power system where agents are power companies investing in new capacity. Our agents form expectations about several unknowns: the level of the future carbon tax, the future power demand and the future mix of energy technologies that a new power plant will have to compete with. The agents can choose to invest in wind, solar PV, fossil fuel based power technologies and a generic carbon-neutral baseload technology (i.e., a technology with no carbon emissions and a high capital cost and low operating cost such as nuclear power.) There is no storage technology in the model. We impose a carbon tax 
on our power system and observe how the competition between these technologies plays out. We compare our ABM with an optimization model that maximizes the sum of consumer and producer surplus, given the same discount rate as the agents use.

We find that the use of wind initially increases with the carbon tax, but that (perhaps somewhat counterintuitively) the use of wind drops when the carbon tax increases beyond a certain level. For high carbon taxes, the carbon-neutral baseload technology expands and eventually outcompetes a certain fraction of the total wind capacity. The reason for this, and under which circumstances it holds, is thoroughly investigated in the paper.

Our conclusions depend on the technoeconomic assumptions made. For instance, we have not assumed that any storage technologies are available. Haegel et al. [45] assume that an electricity storage price of only $\$ 0.025 / \mathrm{kWh}$ per round trip (chargedischarge) could be reached by 2030 . The introduction of low cost storage technologies in our model could significantly affect our results. In future work we plan to include storage technologies in order to explore such scenarios.

In the ABM our agents have limited foresight. We find that agents initially underinvest in wind when the carbon tax is introduced but later over-invest in wind as the tax increases further, compared to the optimal solution.

We also find that optimization models may overestimate the $\mathrm{CO}_{2}$ reduction associated with a given carbon tax. There are two main reasons for this. (1) Optimization models implicitly assume that all actors have perfect foresight and (2) they typically use a social discount rate that is lower than what real actors actually use. Out of these two it is the second feature that has the largest effect in our study. The discount rate affects the relative cost of different technologies and a lower discount rate favors capital intensive but low emitting technologies such as solar, wind and nuclear. This mechanism is well known but is rarely pointed out by researchers who use optimization models.

We also find that higher average electricity prices may be expected when an introduction of VRE is driven by an increasing carbon tax. This stands in contrast to results obtained in many other papers on the impact of a large scale introduction of VRE on electricity prices (see e.g. [31-39]). However, these papers focus on cases where wind and solar power is introduced through a support scheme rather than a price on carbon.

Acknowledgements Financial support from the Swedish Energy Agency and Carl Bennet AB is gratefully acknowledged. We would also like to thank Fredrik Hedenus, Olof Johansson-Stenman, Anders Sandoff and Stefan Park for discussions and valuable input, and Lina Reichenberg for supplying data on German electricity demand, wind speeds and solar insolation. Finally, we are very grateful for valuable comments from an anonymous reviewer, which have helped us to improve the paper.

Open Access This article is distributed under the terms of the Creative Commons Attribution 4.0 International License (http://creativecommons.org/licenses/by/4.0/), which permits unrestricted use, distribution, and reproduction in any medium, provided you give appropriate credit to the original author(s) and the source, provide a link to the Creative Commons license, and indicate if changes were made. 


\section{Appendices}

\section{A. Time slicing}

Time slicing is a method of representing intra-annual variability of e.g. renewable energy output and consumer demand in energy systems models. Each time slice aggregates together all hours that fulfill the characteristics of the slice, irrespective of when during the year they occur. Lehtveer et al. [46] evaluate time slicing in two dimensions, wind and solar PV output, and find that as little as $3^{2}$ to $4^{2}$ slices may provide acceptable solutions for large integrated assessment-type models. In their study they assume that solar output and electricity demand are correlated. Since our models are relatively simple with low computational requirements we can afford to increase the number of slices by including demand as a third dimension. We have chosen to divide each dimension into four different levels: low, medium-low, medium-high and high (see Fig. 14), so that we get $4^{3}=64$ time slices. Table 2 shows the number of hours in a year that is allocated to each time slice.

The top row of Fig. 14 shows the load duration curves for solar PV and wind as shares of total capacity, and demand, that we use as input data to our time slicing. The

Table 2 The time slicing is done in three dimensions: demand reference level $q_{0}$ (see Eq. 1), wind output and solar output

\begin{tabular}{|c|c|c|c|c|}
\hline & Low wind & Medium-low wind & Medium-high wind & High wind \\
\hline \multicolumn{5}{|l|}{ Low solar } \\
\hline Low $q_{0}$ & 28 & 146 & 65 & 25 \\
\hline Med.-low $q_{0}$ & 256 & 1027 & 751 & 283 \\
\hline Med.-high $q_{0}$ & 153 & 645 & 434 & 255 \\
\hline High $q_{0}$ & 16 & 63 & 70 & 73 \\
\hline \multicolumn{5}{|l|}{ Medium-low solar } \\
\hline Low $q_{0}$ & 34 & 128 & 53 & 21 \\
\hline Med.-low $q_{0}$ & 104 & 398 & 213 & 72 \\
\hline Med.-High $q_{0}$ & 59 & 268 & 235 & 119 \\
\hline High $q_{0}$ & 10 & 34 & 33 & 46 \\
\hline \multicolumn{5}{|c|}{ Medium-high solar } \\
\hline Low $q_{0}$ & 24 & 35 & 19 & 7 \\
\hline Med.-low $q_{0}$ & 124 & 227 & 163 & 81 \\
\hline Med.-high $q_{0}$ & 264 & 703 & 419 & 138 \\
\hline High $q_{0}$ & 17 & 52 & 89 & 53 \\
\hline \multicolumn{5}{|l|}{ High solar } \\
\hline Low $q_{0}$ & 0 & 0 & 0 & 0 \\
\hline Med.-low $q_{0}$ & 23 & 15 & 15 & 1 \\
\hline Med.-high $q_{0}$ & 48 & 102 & 10 & 8 \\
\hline High $q_{0}$ & 1 & 3 & 2 & 0 \\
\hline
\end{tabular}

The table shows the number of hours that is allocated into each time slice 


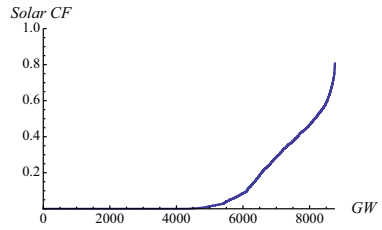

(a) Solar output, original

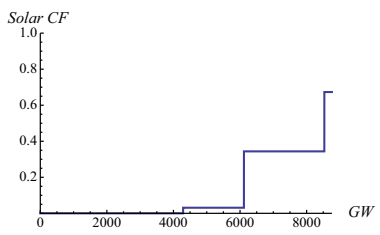

(d) Solar output

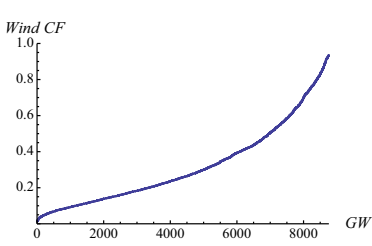

(b) Wind output, original

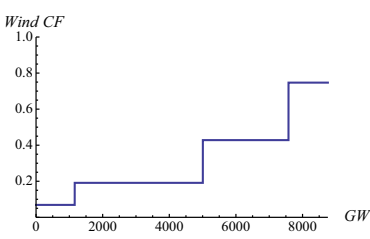

(e) Wind output

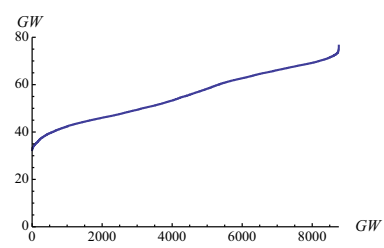

(c) Demand, original

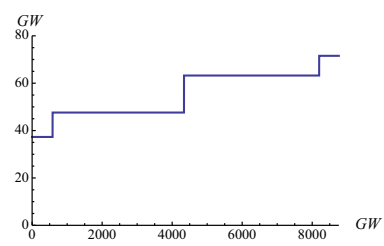

(f) Demand

Fig. 14 Load duration curves for solar PV, wind and demand. The top row shows the original data that we use. The bottom row shows the data when we have converted each of the data series into four discrete levels. a Solar output, original. b Wind output, original. c Demand, original. d Solar output. e Wind output. f Demand

bottom row shows the levels and duration of solar and wind output, and demand, after the time slicing is done.

\section{B. The optimization model}

The optimization model represents the same stylized power system as the ABM, with one single node, no electricity storage and no trade with other regions. As in the ABM, variability of supply and demand is explicitly represented as time slices over the year, with each time slice characterized by a certain wind and solar availability as well as a certain shift in the demand schedule. The model runs in time steps of 5 years and maximizes the discounted sum of consumer and producer surplus over the modeling period of 70 years. The consumer surplus, $C S(t)$, in time step $t$ is given as

$$
C S(t)=K-\sum_{\tau} \frac{p_{\tau}(t) q_{\tau}(t)}{1+\epsilon}
$$

where $K$ is a constant, $p_{\tau}$ is the electricity price [€/MWh] in time slice $\tau, q_{\tau}$ is the quantity electricity consumed [MWh] in time slice $\tau$, and $\epsilon$ is the short term price elasticity of demand (see Sect. 2.1). The producer surplus $P S(t)$ in time step $t$ is

$$
P S(t)=\sum_{i}\left(\sum_{\tau}\left(p_{\tau}(t)-v_{i}-e_{i} \operatorname{tax}(t)\right) q_{i, \tau}(t)-C_{i}(t) I_{i} C R F_{i}\right)
$$

where $v_{i}$ is the variable operating cost of technology $i, e_{i}$ is the emission intensity of technology $i$ and $q_{i, \tau}$ is the amount of electricity sold by technology $i$ in time slice $\tau$. 
$C_{i}(t)$ is the installed capacity [MW] of technology $i$ in time step $t, I_{i}$ is the investment cost [€/MW] of technology $i$ and $C R F_{i}$ is the capital recovery factor of technology $i$.

Since we use 5-year times steps, we assume that system characteristics are constant over a 5-year period around the year of the considered time steps. (For the last time step in the optimization, though, only the two immediately preceding years are included together with the final year of the optimization period.) The optimization problem can then be formulated as

$$
\max _{X(t)} \sum_{t=1}^{T-1} d_{5} \beta^{5 \cdot t} S(t)+d_{3} \beta^{5 \cdot T} S(T)
$$

where $\beta=1 /(1+r)$, the number of time steps $T=14$ and $S(t)=C S(t)+P S(t)$ is the sum of consumer and producer surplus. The factor $d_{5}=\beta^{-2}+\beta^{-1}+1+\beta+\beta^{2}$ corrects for the different discount factors internally within a 5-year step, and similarly does $d_{3}=\beta^{-2}+\beta^{-1}+1$ for the last time step including the three years at the end. The annual investments $X(t)$, composed by investments $x_{i}(t)$ in the different power technologies $i$ in MW, are the independent variables that determine $C S$ and $P S$. The capacity of the initial plants in time step 0 , as well as the carbon tax throughout the modeling period are given. The discount rate $r$ is $4 \%$ (if nothing else is stated) and represents the social rate of discount. As in the ABM the initial plants have a uniform distribution of remaining plant lifetimes. The model is non-linear due to the elastic demand in each hour given by Eq. (1). We solve the optimization problem with a local search heuristic, starting from a feasible solution. A heuristic can in general not guarantee to identify the optimal solution. We do note, however, that the optimization model finds the same steady state as the ABM as long as the same discount rate is used.

\section{The steady state solution}

Both the ABM and the optimization model describe the development of a power system over time as it undergoes a transition. The models are generally not in steady state in

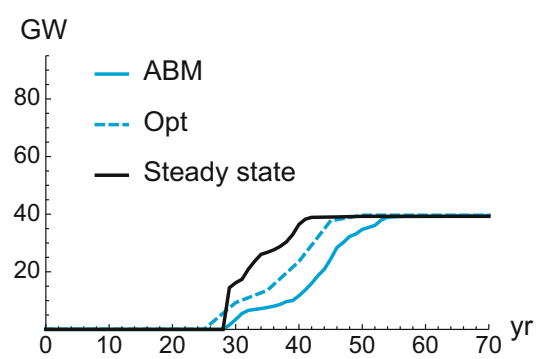

(a) CN-Baseload

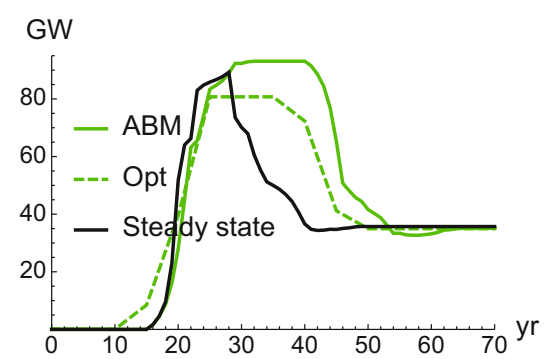

(b) Wind

Fig. 15 Installed capacity by year in the ABM and the optimization model of CN-Baseload (a), wind power (b) and solar PV (c). The solid black lines indicate the steady state solution for the tax level associated with each year. a CN-Baseload. b Wind 
any particular time step, except in the initial and final years when all costs are constant over time. Here we estimate the steady state capacities for each tax level. Figure 15 shows the steady state solution of CN-Baseload and wind for the tax level associated with each year. The capacity trajectories of the ABM and the optimization model are also included for comparison. For the ABM and the optimization model we show the solution for each year over time but for the steady state solution we show the level of installed capacity that our models approach over time if the tax remains constant.

\section{References}

1. Azar, C., Lindgren, K., Andersson, B.A.: Global energy scenarios meeting stringent $\mathrm{CO}_{2}$ constraintscost-effective fuel choices in the transportation sector. Energy Policy 31(10), 961-976 (2003)

2. Blok, K., de Jager, D., Hendriks, C., Kouvaritakis, N., Mantzos, L.: Economic evaluation of sectoral emission reduction objectives for climate change-comparison of topdown and bottom-up analysis of emission reduction opportunities for $\mathrm{CO}_{2}$ in the European Union. Ecofys, AEA and NTUA, Report for European Commission, DG Environment, Brussels, September (2001)

3. Blesl, M., Kober, T., Kuder, R., Bruchof, D.: Implications of different climate protection regimes for the EU-27 and its member states through 2050. Clim. Policy 12(3), 301-319 (2012)

4. Haller, M., Ludig, S., Bauer, N.: Decarbonization scenarios for the EU and MENA power system: considering spatial distribution and short term dynamics of renewable generation. Energy Policy 47, 282-290 (2012)

5. Trutnevyte, E.: Does cost optimization approximate the real-world energy transition? Energy 106, 182-193 (2016)

6. Tesfatsion, L.: Electric power markets in transition: agent-based modeling tools for transactive energy support. Handbook of Computational Economics, vol. 4. Elsevier, Oxford (2017)

7. Murphy, F.H., Smeers, Y.: Generation capacity expansion in imperfectly competitive restructured electricity markets. Oper. Res. 53(4), 646-661 (2005)

8. Browne, O., Poletti, S., Young, D., et al.: How does market power affect the impact of large scale wind investment in energy only wholesale electricity markets? Energy Policy 87(C), 17-27 (2015)

9. Sun, J., Tesfatsion, L.: Dynamic testing of wholesale power market designs: an open-source agent-based framework. Comput. Econ. 30(3), 291-327 (2007)

10. Nicolaisen, J., Petrov, V., Tesfatsion, L.: Market power and efficiency in a computational electricity market with discriminatory double-auction pricing. IEEE Trans. Evolut. Comput. 5(5), 504-523 (2001)

11. De Vries, L.J., Chappin, E.J., Richstein, J.C.: EMLab-Generation, an experimental environment for electricity policy analysis. TU Delft (2013)

12. Richstein, J.C., Chappin, E.J., de Vries, L.J.: Cross-border electricity market effects due to price caps in an emission trading system: an agent-based approach. Energy Policy 71, 139-158 (2014)

13. Hirth, L.: The optimal share of variable renewables. Energy J. 36(1), 127-162 (2015)

14. De Sisternes, F.J., Jenkins, J.D., Botterud, A.: The value of energy storage in decarbonizing the electricity sector. Appl. Energy 175, 368-379 (2016)

15. Gilbert, N.: Agent-Based Models, vol. 153. Sage, Thousand Oaks (2008)

16. Farmer, J.D., Hepburn, C., Mealy, P., Teytelboym, A.: A third wave in the economics of climate change. Environ. Resour. Econ. 62(2), 329-357 (2015)

17. Stern, N.: Economics: current climate models are grossly misleading. Nature 530(7591), 407-409 (2016)

18. Ranci, P., Cervigni, G.: The Economics of Electricity Markets: Theory and Policy. Edward Elgar, Cheltenham (2013)

19. Tietjen, O., Pahle, M., Fuss, S.: Investment risks in power generation: a comparison of fossil fuel and renewable energy dominated markets. Energy Econ. 58, 174-185 (2016)

20. Noothout, P., de Jager, D., Tesnière, L., van Rooijen, S., Karypidis, N., Brückmann, R., Jirouš, F., Breitschopf, B., Angelopoulos, D., Doukas, H., et al.: The impact of risks in renewable energy investments and the role of smart policies. DiaCore report (2016) 
21. Energimarknadsinspektionen: Ökad andel variabel elproduktion Effekter på priser och producenters investeringsincitament. Swedish Energy Markets Inspectorate (2016). http://www.ei.se. Accessed 29 Mar 2017

22. Brealey, R.A., Myers, S.C., Allen, F., Mohanty, P.: Principles of Corporate Finance. Tata McGraw-Hill Education, New York CIty (2011)

23. Thorson, L., Johansson, V.: Modelling of wind power-a techno-economic analysis of wind turbine configuration. Master's thesis, Chalmers University of Technology (2016)

24. Norwood, Z., Nyholm, E., Otanicar, T., Johnsson, F.: A geospatial comparison of distributed solar heat and power in Europe and the US. PloS One 9(12), e112442 (2014)

25. IEA Wind TCP 2015 Annual Report: PWT Communications. LLC, Boulder, CO. (2016). http://www. ieawind.org

26. Iyer, G.C., Clarke, L.E., Edmonds, J.A., Flannery, B.P., Hultman, N.E., McJeon, H.C., Victor, D.G.: Improved representation of investment decisions in assessments of $\mathrm{CO}_{2}$ mitigation. Nat. Clim. Change 5(5), 436-440 (2015)

27. Hirth, L., Steckel, J.C.: The role of capital costs in decarbonizing the electricity sector. Environ. Res. Lett. 11(11), 114,010 (2016)

28. Kolstad, C., Urama, K., Broome, J., Bruvoll, A., Cariño-Olvera, M., Fullerton, D., Gollier, C., Hanemann, W.M., Hassan, R., Jotzo, F., et al.: Social, economic and ethical concepts and methods. In: Climate Change 2014: Mitigation of Climate Change, pp. 173-248 (2014)

29. Zhuang, J., Liang, Z., Lin, T., De Guzman, F.: Theory and practice in the choice of social discount rate for cost-benefit analysis: a survey. ERD working paper series (2007)

30. Hermelink, A.H., de Jager, D.: Evaluating our future. The crucial role of discount rates in European Commission energy system modelling The European Council for an Energy Efficient Economy (eceee) \& Ecofys (2015)

31. Sáenz de Miera, G., del Río González, P., Vizcaíno, I.: Analysing the impact of renewable electricity support schemes on power prices: the case of wind electricity inSpain. Energy Policy 36(9), 3345-3359 (2008)

32. Sensfuß, F., Ragwitz, M., Genoese, M.: The merit-order effect: a detailed analysis of the price effect of renewable electricity generation on spot market prices in Germany. Energy Policy 36(8), 3086-3094 (2008)

33. Woo, C.K., Horowitz, I., Moore, J., Pacheco, A.: The impact of wind generation on the electricity spot-market price level and variance: the Texas experience. Energy Policy 39(7), 3939-3944 (2011)

34. Gil, H.A., Gomez-Quiles, C., Riquelme, J.: Large-scale wind power integration and wholesale electricity trading benefits: estimation via an ex post approach. Energy Policy 41, 849-859 (2012)

35. O'Mahoney, A., Denny, E.: The merit order effect of wind generation on the Irish electricity market (2011). http://mpra.ub.uni-muenchen.de/56043/. Accessed 24 Mar 2017

36. MacCormack, J., Hollis, A., Zareipour, H., Rosehart, W.: The large-scale integration of wind generation: impacts on price, reliability and dispatchable conventional suppliers. Energy Policy 38(7), 3837-3846 (2010)

37. Würzburg, K., Labandeira, X., Linares, P.: Renewable generation and electricity prices: taking stock and new evidence for Germany and Austria. Energy Econ. 40, S159-S171 (2013)

38. Clò, S., Cataldi, A., Zoppoli, P.: The merit-order effect in the Italian power market: the impact of solar and wind generation on national wholesale electricity prices. Energy Policy 77, 79-88 (2015)

39. Dillig, M., Jung, M., Karl, J.: The impact of renewables on electricity prices in Germany-an estimation based on historic spot prices in the years 2011-2013. Renew. Sustain. Energy Rev. 57, 7-15 (2016)

40. Hirth, L.: The market value of variable renewables: the effect of solar wind power variability on their relative price. Energy Econ. 38, 218-236 (2013)

41. Hedenus, F., Azar, C., Lindgren, K.: Induced technological change in a limited foresight optimization model. Energy J. 27, 109-122 (2006)

42. Keppo, I., Strubegger, M.: Short term decisions for long term problems-the effect of foresight on model based energy systems analysis. Energy 35(5), 2033-2042 (2010)

43. Chen, H., Ma, T.: Technology adoption with limited foresight and uncertain technological learning. Eur. J. Oper. Res. 239(1), 266-275 (2014)

44. Welkenhuysen, K., Piessens, K.: The essential role of uncertainty and limited foresight in energy modelling. Self J. Sci. (2017) http://www.sjscience.org/article?id=598. Accessed 11 Mar 2017 
45. Haegel, N.M., Margolis, R., Buonassisi, T., Feldman, D., Froitzheim, A., Garabedian, R., Green, M., Glunz, S., Henning, H.M., Holder, B.: Terawatt-scale photovoltaics: trajectories and challenges. Science 356(6334), 141-143 (2017)

46. Lehtveer, M., Mattsson, N., Hedenus, F.: Using resource based slicing to capture the intermittency of variable renewables in energy system models. Energy Strat. Rev. 18, 73-84 (2017) 